\title{
The Association Between Different Obesity Phenotypes and Liver Fibrosis Scores in Elderly Individuals with Fatty Liver in Taiwan
}

This article was published in the following Dove Press journal: Diabetes, Metabolic Syndrome and Obesity: Targets and Therapy

\author{
Yu-Shan Lee (D)' \\ Lee-Ching Hwang (iD) ${ }^{1,2}$ \\ Hsin-Yin Hsu id ${ }^{\prime}$ \\ Meng-Ting Tsou (D) ${ }^{1,3}$ \\ 'Department of Family Medicine, Mackay \\ Memorial Hospital, Taipei, Taiwan; \\ ${ }^{2}$ Department of Medicine, MacKay \\ Medical College, New Taipei City, Taiwan; \\ ${ }^{3}$ Mackay Junior College of Medicine, \\ Nursing, and Management, Taipei, Taiwan
}

Purpose: To examine the association between different phenotypes of obesity or metabolic syndromes and liver fibrosis score in a Taiwanese elderly population with fatty liver.

Patients and Methods: This cross-sectional study included 1817 participants aged $\geq 65$ years with fatty liver diagnosed by sonography. We used ethnicity-specific criteria for body mass index and metabolic syndrome, and to define obesity phenotypes as metabolically healthy non-obese (MHNO), metabolically unhealthy non-obese (MUNO), metabolically healthy obese (MHO), and metabolically unhealthy obese (MUO). Correlated fibrosis severity was calculated using the nonalcoholic fatty liver disease (NAFLD) fibrosis score (NFS) and Fibrosis-4 (FIB-4). Fibrosis severity was divided into two categories according to NFS (no-to-mild fibrosis and advanced fibrosis, defined as NFS $\leq 0.676$ and $>0.676$, respectively) and FIB-4 score (no-to-mild fibrosis and advanced fibrosis, defined as FIB-4 score $\leq 2.67$ and $>2.67$, respectively).

Results: Compared with that in the MHNO group, the associated risk (odds ratio [OR], 95\% confidence interval [CI]) of advanced fibrosis by NFS was 2.43 (1.50-3.93), 2.35 (1.25-4.41), and 6.11 (3.90-9.59), whereas that of advanced fibrosis by FIB-4 score was 1.34 (0.83-2.18), 2.37 (1.36-4.13), and 1.38 (0.82-2.31) in the MUNO, MHO, and MUO groups, respectively. Conclusion: Both metabolic syndrome and obesity were positively associated with more advanced fibrosis according to NFS. The detrimental effect of obesity appears to be more than metabolic abnormalities per se in the elderly with more advanced fibrosis severity according to the FIB-4 score.

Keywords: nonalcoholic fatty liver disease, obese phenotype, elderly population, NAFLD fibrosis score, Fibrosis-4

\section{Introduction}

Nonalcoholic fatty liver disease (NAFLD) is one of the most common liver disorders worldwide. Its prevalence ranges from $24.0 \%$ to $46.0 \%$ in the general population across western countries and from $7.9 \%$ to $54 \%$ in Asian countries. ${ }^{1}$ In Taiwan, its prevalence is approximately $11.5-41.0 \%,{ }^{2,3}$ but one study has disclosed a higher rate $(50.1 \%)$ in the elderly population. ${ }^{4}$

NAFLD is characterized by excess fat in the liver independent of secondary causes of fat accumulation in the liver. Approximately $23-44 \%$ of the patients with NAFLD have nonalcoholic steatohepatitis (NASH), 5,6 a more rapidly progressive form, ${ }^{7}$ and $37-41 \%$ of the patients with NASH develop progressive fibrosis. ${ }^{8,9}$ Besides, about $10-20 \%$ of the patients with NASH progress to cirrhosis within 10 years, whereas the cumulative incidence of hepatocellular carcinoma (HCC) in
Correspondence: Meng-Ting Tsou Department of Family Medicine, Mackay Memorial Hospital, No. 92, Sec. 2,

Zhongshan N. Road, Taipei City, 10449 Taiwan, R.O.C.

Tel +886225433535 (Ext. 2 I 3 I or 2 I32)

Fax +886225433642

Email mttsou@gmail.com
Diabetes, Metabolic Syndrome and Obesity: Targets and Therapy 202 I:14 |473-|483

mit your manuscrip (c) (i) (5) 2021 Lee et al. This work is published and licensed by Dove Medical Press Limited. The full terms of this license are available at https://www.dovepress.com/terms.php CY ${ }_{\mathrm{NC}}$ and incorporate the Creative Commons Attribution - Non Commercial (unported, v3.0) License (http://creativecommons.org/licenses/by-nc/3.0/). By accessing the work you hereby accept the Terms. Non-commercial uses of the work are permitted without any further permission from Dove Medical Press Limited, provided the work is properly attributed. For permission for commercial use of this work, please see paragraphs 4.2 and 5 of our Terms (https://www.dovepress.com/terms.php). 
NASH-related cirrhosis ranges from $2.4 \%$ to $12 \%$ within 3-7 years. ${ }^{10,11}$ The presence and severity of fibrosis predict patients' overall and liver-related mortality. ${ }^{12,13}$ Although liver biopsy is the gold standard procedure for assessing NAFLD severity, it is invasive, with the risk of periprocedural complications and mortality. ${ }^{14}$ In addition, performing liver biopsy for all individuals having or being at risk with NASH is unrealistic. Therefore, noninvasive methods for liver fibrosis assessment have been developed. NAFLD fibrosis score (NFS) and Fibrosis-4 score (FIB-4) are among the most common noninvasive markers of fibrosis with acceptable accuracy to detect advanced fibrosis in NAFLD. ${ }^{14-17}$ Advance fibrosis can be diagnosed with high accuracy (positive predictive value [PPV], 90\%) using the high cutoff score $(>0.676)$ in NFS and a PPV of $80 \%$ using the high cutoff score $(>2.67)$ for FIB-4. ${ }^{14-16}$

Risk factors for NAFLD include obesity, type 2 diabetes mellitus (T2DM), hypertension, and dyslipidemia, which are often categorized together as the insulin resistance syndrome or metabolic syndrome. ${ }^{18}$ With the progressive epidemic of obesity and metabolic syndromes, NAFLD prevalence is expected to rise. In a crosssectional survey, Fan et al reported that fatty liver was 11.6-fold (95\% confidence interval [CI], 8.2-16.5) higher in patients with obesity and was increased by 39.3-fold (95\% CI, 17.8-87.1) in patients with metabolic syndrome. ${ }^{19,20}$ Moreover, evidence showed that metabolic healthy obesity (MHO) may significantly be associated with increased risk of NAFLD and advanced fibrosis, with risks intermediate between metabolic healthy nonobese (MHNO) and metabolic unhealthy obese (MUO). ${ }^{21}$ A multicenter cross-sectional study in Spain showed that $\mathrm{NASH}$ was more frequent in metabolic unhealthy individuals (metabolic unhealthy non-obese [MUNO], 55.8\%; MUO, 57.8\%) than metabolic healthy individuals (MHNO, 26.9\%; MHO, 42.4\%) ${ }^{22}$ and another study in Europe reported that fibrosis increased progressively with numbers of metabolic alteration. ${ }^{23}$

In addition, accumulating evidence suggest that NAFLD may be both a consequence and a cause of various components of metabolic syndrome, particularly T2DM and to a lesser extent, hypertension. ${ }^{24}$ A large Taiwanese population study comprised 132,377 nondiabetic individuals with two or more health examinations during 1996-2014, showed that ultrasound-diagnosed NAFLD was significantly associated with two-fold increase in incident T2DM. ${ }^{25}$ Another retrospective cohort study of 11,448 participants with 5-year follow-up in South Korea revealed that development of NAFLD was associated with increased risk of incident hypertension (odds ratio [OR], 1.36 [1.1-1.7]) compared with resolution of NAFLD at follow-up. ${ }^{26}$

Furthermore, patients with NAFLD at an advanced age are more at risk for cirrhosis and even a higher mortality. ${ }^{27}$ In a Japanese retrospective study, severe fibrosis was significantly more prevalent in older patients ( $\geq 60$ years old) than in younger patients $(29 \%$ vs $53 \%, p=0.02) .{ }^{28}$ Therefore, health professionals must know how to identify NAFLD in elderly individuals. However, NAFLD is often overlooked, with few studies of NAFLD focusing on the elderly. The insight into whether obesity itself or metabolic abnormalities have more influence on NAFLD remains unconfirmed. Additionally, sex differences do exist in the prevalence of NAFLD and liver fibrosis severity, which are higher in men than women during the reproductive age but changes after menopause. ${ }^{29}$ Nevertheless, the connection is not fully understood and more studies are needed to fill in the research gap. With the elderly as the major group for metabolic syndrome and obesity, we are interested in the relationship between NAFLD and fibrosis risks. Hence, this cross-sectional study aimed to investigate the association between the different phenotypes of obesity or metabolic syndrome and the NFS and FIB-4 score in a Taiwanese elderly population with fatty liver.

\section{Materials and Methods Study Population}

A total of 17,115 participants (aged $\geq 65$ years) underwent health examinations in Taipei Mackey Memorial Hospital between 2008 and 2018. Among them, 4105 participants with fatty liver diagnosed by abdominal sonography were included in this study.

However, we excluded those who had chronic viral hepatitis, which was defined in this study as having a history of hepatitis B virus or hepatitis $\mathrm{C}$ virus or having tested positive for hepatitis B antigen or hepatitis $\mathrm{C}$ antibodies documented in medical records; those who reported heavy drinking upon examination, defined as more than 4 drinks on any day for men or more than 3 drinks for women according to National Institute on Alcohol Abuse and Alcoholism; those with incomplete data on fasting glucose level, platelet count, triglyceride (TG), high- 
density lipoprotein cholesterol (HDL-C), albumin, AST and ALT; and those with repeated visits (Figure 1).

The study protocol was evaluated and approved by the Human Research Ethics Committee of Mackay Memorial Hospital (project research number 18MMHIS137).

\section{Data Collection and Measurements}

To obtain medical history, all participants completed a questionnaire that included demographic characteristics, personal health history, current medication use, smoking habit, and alcohol consumption. Here, they self-reported their current smoking status as either nonsmoker, social smoker, or current smoker, and their alcohol consumption as either no drinking, social drinking, or heavy drinking. We also collected data on blood pressure (BP), body weight, height, waist circumference, and other biomarker measurements including complete blood counts, liver and renal function tests, lipid panels, glucose, and albumin. The Friedewald equation and Martin/Hopkins density lipoprotein cholesterol (LDL-C) method (if $\mathrm{TG} \geq 400$ ) were used in those without serum levels for LDL-C. ${ }^{30,31}$ Furthermore, well-trained gastroenterologists performed abdominal sonography to categorize fatty liver into two groups, namely, mild and moderate-to-severe groups. ${ }^{32}$

\section{Assessment of Obesity and Metabolic Health Status}

Obesity and metabolic syndrome were defined using the ethnic-specific criteria made by Taiwan Health Promotion Administration, Ministry of Health and Welfare. BMI was

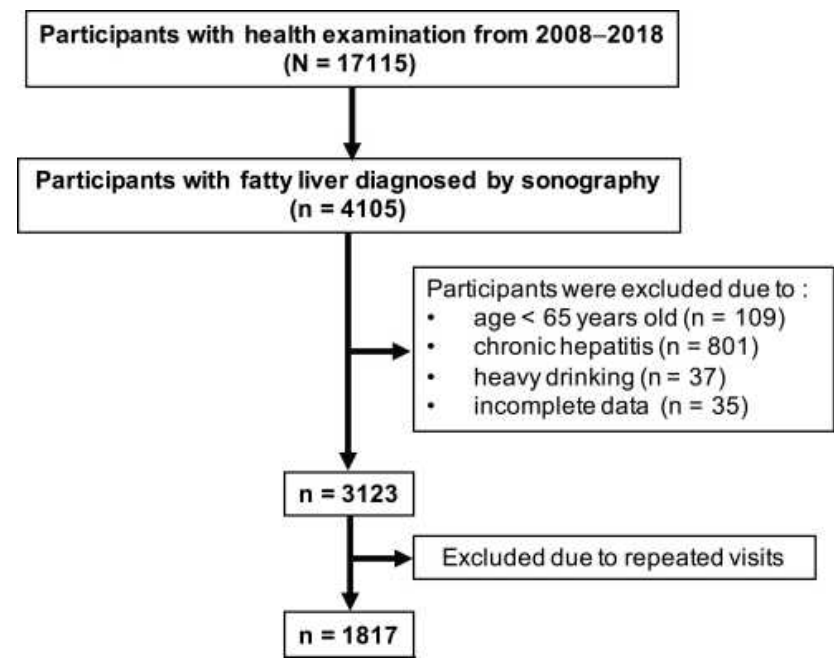

Figure I Flow chart of evaluated participants with fatty liver. calculated as weight in kilograms divided by the square of the participants' height in meters. Obesity was defined as BMI $\geq 27$, and non-obese as BMI $<27 .{ }^{33}$ Meanwhile, metabolic syndrome was diagnosed in a participant with three or more of the following criteria: 1) central obesity (abdominal circumference $\geq 90 \mathrm{~cm}$ for men and $\geq 80 \mathrm{~cm}$ for women); 2) elevated serum triglyceride level ( $\geq 150 \mathrm{mg} / \mathrm{dL}$ ) or taking lipid-lowering drugs; 3) decreased HDL cholesterol level ( $<40 \mathrm{mg} / \mathrm{dL}$ for men and $<50 \mathrm{mg} / \mathrm{dL}$ for women); 4) elevated BP (systolic $\mathrm{BP} \geq 130 \mathrm{mmHg}$ or diastolic $\mathrm{BP} \geq 85$ $\mathrm{mmHg}$ ) or history of hypertension or taking antihypertensive drug; and 5) elevated fasting glucose level ( $\geq 100 \mathrm{mg} / \mathrm{dL}$ ) or history of diabetes mellitus or taking hypoglycemic agents. Then, using the definition above, we categorized the participants into following four phenotypes: 1) metabolically healthy non-obese (MHNO), 2) metabolically unhealthy non-obese (MUNO), 3) metabolically healthy obese (MHO), and 4) metabolically unhealthy obese (MUO). ${ }^{34,35}$

\section{Noninvasive Markers of Fibrosis and Fibrosis Severity Categories}

We used NFS and FIB-4 to calculate correlated fibrosis severity. ${ }^{15,16}$ NFS and FIB-4 were calculated using the following equations: NFS $=(-1.675)+0.037-$ age (years) $+0.094-$ BMI $\left(\mathrm{kg} / \mathrm{m}^{2}\right)+1.13 \times$ impaired fasting glucose $(\mathrm{IFG}) /$ diabetes $($ yes $=1$, no $=0)+0.99 \times$ AST $/$ ALT ratio $-0.013 \times$ platelet count $\left(\times 10^{9} / \mathrm{L}\right)-0.66 \times$ albumin $(\mathrm{g} / \mathrm{dL})$; and FIB-4 = age $\times$ AST $(\mathrm{IU} / \mathrm{L}) /$ platelet count $\left(\times 10^{9} / \mathrm{L}\right) \times \sqrt{ }$ ALT $(\mathrm{IU} / \mathrm{L})$.

For each score, advanced fibrosis was defined using previously published cutoff values. ${ }^{14-16,36}$ The fibrosis severity defined by NFS was categorized into two groups: no-to-mild fibrosis $(\leq 0.676)$ and advanced fibrosis $(>0.676)$. Fibrosis severity divided by the FIB-4 score used the cutoff value of $\leq 2.67$ for no-to-mild fibrosis and $>2.67$ for advanced fibrosis.

\section{Statistical Analysis}

We used descriptive statistics to summarize the characteristics of the participants according to the four phenotypes (MHNO, MUNO, MHO, and MUO). These descriptive statistics were expressed as mean $\pm \mathrm{SD}$ for continuous variables and percentages for categorical variables. Significant differences in the characteristics among the four phenotypes were determined by analysis of variance tests for the continuous variables and chisquare tests for categorical variables.

Using logistic regression and $\mathrm{MHNO}$ as control group, we calculated the OR and $95 \% \mathrm{CI}$ to describe the 
association between obese phenotypes and advanced fibrosis. Potential confounders were adjusted via the following three models: Model 1, sex; Model 2, factors in Model 1 plus smoking status and alcohol consumption; Model 3, factors in Model 2 plus fasting glucose, total cholesterol (TC), TG and HDL. Subgroup analysis stratified by sex was performed and adjusted for potential confounders as above.

All statistical data were analyzed using the SPSS version 24.0 software (IBM Corp., Armonk, NY). A $p$ value of $<0.05$ was considered to be statistically significant.

\section{Results}

A total of 1817 participants (587 males and 1230 females, aged $72.9 \pm 5.9$ years) were analyzed. Among them, 580, 671, 131, and 435 were classified into MHNO, MUNO, MHO, and MUO groups, respectively. Table 1 presents the characteristics of study participants stratified by obesity phenotypes and characteristics of sex-based subgroups are shown in Supplemental Table 2.1, 2.2 and Supplemental Table 3.1, 3.2. The percentage for moderateto-severe fatty liver diagnosed by sonography was $5.0 \%$, $13.0 \%, 18.3 \%$, and $31.3 \%$ in the MHNO, MUNO, MHO, and MUO group $(p<0.05)$. Furthermore, the percentage for advanced fibrosis according to the NFS score (NFS $>$ 0.676 ) was $6.7 \%, 12.8 \%, 13.0 \%, 30.3 \%$ in the MHNO, MUNO, MHO, and MUO groups $(p<0.05)$, with an average NFS score of $-0.840( \pm 1.0),-0.361( \pm 1.0)$, $-0.292( \pm 0.9)$, and $0.176( \pm 0.4)(p<0.05)$, respectively. Meanwhile, the percentage for advanced fibrosis according to the FIB-4 score (FIB-4 >2.67) was $8.3 \%, 9.5 \%, 16.8 \%$, and $9.7 \%$ in the MHNO, MUNO, MHO and MUO groups $(p=0.031)$, with an average FIB-4 score of $1.84( \pm 0.8)$, $1.83( \pm 0.7), 1.82( \pm 0.9)$, and $1.98( \pm 0.7)(p=0.20)$, respectively. Compared with the MHNO group, the MUNO, MHO, and MUO groups were more likely to have moderate-to-severe fatty liver (MUNO: OR, 2.83 [1.83-4.37]; MHO: OR, 4.30 [2.41-7.68]; MUO: OR, 8.64 [5.65-13.22]) (Supplemental Table 1.1, 1.2).

Table 2 shows the comparison of advanced fibrosis categorized by the NFS score (NFS $>0.676$ ) between the MUNO, $\mathrm{MHO}$, and MUO groups and the MHNO group by multiple logistic regression analysis. Compared with the MHNO group, the odds of liver fibrosis were significantly higher in MUNO, MHO, and MUO groups (2.03 [1.37-3.02], 2.09 [1.14-3.82], and 6.04 [4.12-8.87], respectively). After we adjusted for covariates such as sex, smoking habit, alcohol consumption, fasting glucose, TC, TG, and $\mathrm{HDL}$, the results were fundamentally the same (MUNO: OR, 2.43 [1.50-3.93]; MHO: OR, 2.35 [1.25-4.41]; MUO: OR, 6.11 [3.90-9.59] compared with the MHNO group). In the subgroup analysis stratified by sex, the results showed that compared with the MHNO group, the odds of liver fibrosis were significantly higher in MUNO and MUO groups in both women (MUNO: OR, 2.05 [1.16-3.62]; MUO: OR, 7.01 [4.04-12.15]) and men (MUNO: OR, 2.73 [1.55-4.81]; MUO: OR, 6.17 [3.52-10.83]) with a positive trend in the MHO group (women: OR, 2.09 [0.87-5.12]; men: OR, 2.23 [0.96-5.21]) (Table 3).

Table 4 demonstrates the comparison of advanced fibrosis categorized by the FIB-4 score (FIB-4 >2.67) between the MUNO, MHO, and MUO groups and the MHNO group by multiple logistic regression analysis. Compared with that in the MHNO group, the odds of advanced fibrosis by the FIB-4 score was 1.16 (0.79-1.72), $2.26(1.31-3.90), 1.18(0.77-1.83)$ in the MUNO, MHO, and MUO groups, respectively. After adjusting potential confounding covariates, we found a positive trend for association, with significant finding in the $\mathrm{MHO}$ group (MUNO: OR, 1.34 [0.83-2.18]; MHO: OR, 2.37 [1.36-4.13]; MUO: OR, 1.38 [0.82-2.31] compared with the MHNO group). When dividing the study population according to sex in the subgroup analysis, the association was stronger in women, with significant finding in the MHO group (MUNO: OR, 1.45 [0.82-2.56]; MHO: OR, 3.84 [1.86-7.95]; MUO: OR, 1.54 [0.83-2.85] compared with the MHNO group), whereas no differences were found in men (Table 5).

\section{Discussion}

In our study, both metabolic syndrome and obesity were positively associated with more advanced fibrosis according to the NFS, which showed significant findings, but only a positive trend was revealed when using the FIB-4 score with significantly positive association in the MHO group. Regardless of the metabolic status, those with obesity had a higher percentage of moderate-to-severe fatty liver diagnosed by sonography. Obesity seemed to have more detrimental effects than metabolic abnormalities per se on the severity of more advanced fibrosis in the elderly according to the FIB-4 score. The effect was even more pronounced in elderly women with a three-fold increase in the MHO group, whereas no statistical difference was observed in men.

Our finding is consistent with those of previous studies, ${ }^{37-39}$ which concluded that metabolically healthy 
Table I Characteristics of Study Participants According to Obesity Phenotypes

\begin{tabular}{|c|c|c|c|c|c|}
\hline & MHNO & MUNO & МнO & MUO & \multirow[t]{2}{*}{$p$ value } \\
\hline & $(n=580)$ & $(n=67 I)$ & $(n=|3|)$ & $(n=435)$ & \\
\hline Age (years) & $72.1 \pm 5.8$ & $73.3 \pm 5.9^{\dagger}$ & $73.0 \pm 5.6$ & $73.2 \pm 6.0^{\dagger}$ & 0.001 \\
\hline Female (\%) & $345(59.5)$ & $499(74.4)$ & $82(62.6)$ & $304(69.9)$ & $<0.001$ \\
\hline BMI $\left(\mathrm{kg} / \mathrm{m}^{2}\right)$ & $23.8 \pm 1.8$ & $24.6 \pm 1.6^{\dagger}$ & $29.1 \pm 2.2^{\dagger \neq}$ & $29.6 \pm 2.4^{t \ddagger}$ & $<0.001$ \\
\hline Waist circumference $(\mathrm{cm})$ & $81.2 \pm 6.7$ & $85.3 \pm 6.5^{\dagger}$ & $92.9 \pm 8.9^{\dagger \neq}$ & $94.2 \pm 9.4^{\dagger \ddagger}$ & $<0.001$ \\
\hline Systolic BP (mmHg) & $134.5 \pm 19.8$ & $14 \mid .5 \pm 20.7^{\dagger}$ & $137.6 \pm 18.4$ & $146.0 \pm 20.0^{\dagger \neq \S}$ & $<0.001$ \\
\hline Diastolic BP (mmHg) & $69.4 \pm 10.6$ & $72.4 \pm 10.4^{\dagger}$ & $71.9 \pm 11.1$ & $74.2 \pm 9.9^{\dagger \ddagger}$ & $<0.001$ \\
\hline Glucose (mg/dL) & $101.7 \pm 18.1$ & $115.4 \pm 26.4^{\dagger}$ & $99.8 \pm 14.5^{\ddagger}$ & $117.3 \pm 23.1^{\dagger \S}$ & $<0.001$ \\
\hline Total cholesterol (mg/dL) & $102.4 \pm 40.0$ & $152.1 \pm 76.3^{\dagger}$ & $98.8 \pm 37.0^{\ddagger}$ & $138.0 \pm 64.0^{\dagger \S}$ & $<0.001$ \\
\hline HDL-C (mg/dL) & $60.0 \pm 14.4$ & $50.7 \pm 13.2^{\dagger}$ & $59.3 \pm 12.2^{\ddagger}$ & $51.9 \pm 11.9^{\dagger \S}$ & $<0.001$ \\
\hline LDL-C (mg/dL) & $118.2 \pm 31.0$ & $112.4 \pm 32.9^{\dagger}$ & $124.3 \pm 32.8^{\ddagger}$ & $112.2 \pm 30.4^{\dagger \S}$ & $<0.001$ \\
\hline Triglyceride (mg/dL) & $102.4 \pm 40.0$ & $152.1 \pm 76.3^{\dagger}$ & $98.8 \pm 37.0^{\ddagger}$ & $138.0 \pm 64.0^{\dagger \neq \S}$ & $<0.001$ \\
\hline AST $(U / L)$ & $23.5 \pm 7.1$ & $24.4 \pm 9.2$ & $25.1 \pm 9.7$ & $25.4 \pm 10.4^{\dagger}$ & 0.013 \\
\hline ALT (U/L) & $21.3 \pm 10.2$ & $23.4 \pm 12.4^{\dagger}$ & $22.5 \pm 11.2$ & $25.3 \pm 14.3^{\dagger}$ & $<0.001$ \\
\hline Albumin (mg/dL) & $4.3 \pm 0.2$ & $4.3 \pm 0.2$ & $4.2 \pm 0.2^{\dagger \ddagger}$ & $4.3 \pm 0.2$ & $<0.001$ \\
\hline Platelet $\left(\times 10^{9} / \mathrm{L}\right)$ & $218.4 \pm 51.1$ & $222.0 \pm 50.6$ & $209.2 \pm 50.0$ & $220.0 \pm 58.5$ & 0.08 \\
\hline Smoking habit & & & & & 0.63 \\
\hline Nonsmoker (\%) & $557(96.0)$ & $648(96.6)$ & $129(98.5)$ & $425(97.7)$ & \\
\hline Social smoker (\%) & $9(1.6)$ & $8(1.2)$ & $0(0)$ & $3(0.7)$ & \\
\hline Current smoker (\%) & $14(2.4)$ & $15(2.2)$ & $2(1.5)$ & $7(1.6)$ & \\
\hline Alcohol consumption & & & & & 0.028 \\
\hline No drinking (\%) & $506(87.2)$ & $617(92.0)$ & $113(86.3)$ & $392(90.1)$ & \\
\hline Social drinking (\%) & $74(12.8)$ & $54(8.0)$ & $18(13.7)$ & $43(9.9)$ & \\
\hline Diabetes mellitus (\%) & $39(6.7)$ & 159 (23.7) & $4(3.1)$ & $119(27.4)$ & $<0.001$ \\
\hline Hypertension (\%) & $214(36.9)$ & 394 (58.7) & $56(42.7)$ & $332(76.3)$ & $<0.001$ \\
\hline Hyperlipidemia (\%) & $62(10.7)$ & $218(32.5)$ & $4(3.1)$ & $143(32.9)$ & $<0.001$ \\
\hline Total numbers of metabolic factors & & & & & $<0.001$ \\
\hline None $(\%)$ & $45(7.8)$ & $0(0)$ & $3(2.3)$ & $0(0)$ & \\
\hline One factor (\%) & $187(32.2)$ & $0(0)$ & $17(13.1)$ & $0(0)$ & \\
\hline Two factors (\%) & $348(60.0)$ & $0(0)$ & III (87.4) & $0(0)$ & \\
\hline Three factors (\%) & $0(0)$ & $360(53.7)$ & $0(0)$ & $198(45.5)$ & \\
\hline Four factors (\%) & $0(0)$ & $226(33.7)$ & $0(0)$ & $161(37.0)$ & \\
\hline Five factors (\%) & $0(0)$ & $88(12.8)$ & $0(0)$ & $85(12.7)$ & \\
\hline Fatty liver & & & & & $<0.001$ \\
\hline Mild (\%) & $55 I(95.0)$ & $584(87.0)$ & $107(8 \mathrm{I} .7)$ & $299(68.7)$ & \\
\hline Moderate-to-severe (\%) & $29(5.0)$ & $87(13.0)$ & $24(18.3)$ & $136(31.3)$ & \\
\hline NFS & $-0.840 \pm 1.0$ & $-0.361 \pm 1.0^{\dagger}$ & $-0.292 \pm 0.9^{\dagger}$ & $0.176 \pm 0.4^{\dagger \neq \S}$ & $<0.001$ \\
\hline NFS category & & & & & $<0.001$ \\
\hline No-mild fibrosis (NFS $\leq$ 0.676) (\%) & $54 \mid(93.3)$ & $585(87.2)$ & II4 (87.0) & $303(69.7)$ & \\
\hline Advanced fibrosis (NFS > 0.676) $(\%)$ & $39(6.7)$ & $86(12.8)$ & $17(13.0)$ & $132(30.3)$ & \\
\hline FIB-4 & $1.84 \pm 0.8$ & $1.83 \pm 0.7$ & $1.82 \pm 0.9$ & $1.98 \pm 0.7$ & 0.20 \\
\hline
\end{tabular}

(Continued) 
Table I (Continued).

\begin{tabular}{|c|c|c|c|c|c|}
\hline & MHNO & MUNO & МHO & MUO & \multirow[t]{2}{*}{$p$ value } \\
\hline & $(n=580)$ & $(n=67 I)$ & $(n=|3|)$ & $(n=435)$ & \\
\hline FIB-4 category & & & & & 0.031 \\
\hline No-mild fibrosis (FIB-4 $\leq 2.67$ ) (\%) & $532(91.7)$ & $607(90.5)$ & $109(83.2)$ & $393(90.3)$ & \\
\hline Advanced fibrosis (FIB-4 > 2.67) (\%) & $48(8.3)$ & $64(9.5)$ & $22(16.8)$ & $42(9.7)$ & \\
\hline
\end{tabular}

Notes: Data are presented as number (percentage) or mean \pm standard deviation; p-values calculated using chi-square tests for categorical variables and analysis of variance tests for the continuous variables; ${ }^{\dagger}$ different from MHNO phenotype $(p<0.05)$; ${ }^{\ddagger}$ different from MUNO phenotypes $(p<0.05)$; ${ }^{\S}$ different from MHO phenotype $(p<0.05)$. Abbreviations: BMI, body mass index; BP, blood pressure; HDL-C, high-density lipoprotein cholesterol; LDL-C, low-density lipoprotein cholesterol; AST, aspartate aminotransaminase; ALT, alanine transaminase; NFS, NAFLD fibrosis score; FIB-4, fibrosis-4 score; MHNO, metabolically healthy non-obese; MUNO, metabolically unhealthy non-obese; MHO, metabolically healthy obese; MUO, metabolically unhealthy obese.

Table 2 Univariate Analysis and Multivariate Analysis for Advanced Fibrosis Using NFS (>0.676) According to Obese Phenotypes

\begin{tabular}{|l|l|l|l|l|}
\hline & MHNO & MUNO & MHO & MUO \\
\cline { 2 - 5 } & OR (95\% Cl) & OR (95\% Cl) & OR (95\% Cl) & OR (95\% CI) \\
\hline NFS category & I (reference) & $2.03(1.37-3.02)^{*}$ & $2.09(1.14-3.82)^{*}$ & $6.04(4.12-8.87)^{*}$ \\
Model I & I (reference) & $2.37(1.58-3.56)^{*}$ & $2.16(1.18-3.98)^{*}$ & $6.59(4.46-9.75)^{*}$ \\
Model 2 & I (reference) & $2.39(1.59-3.6 I)^{*}$ & $2.13(1.15-3.93)^{*}$ & $6.58(4.44-9.74)^{*}$ \\
Model 3 & I (reference) & $2.43(1.50-3.93)^{*}$ & $2.35(1.25-4.4 I .)^{*}$ & $6.11(3.90-9.59)^{*}$ \\
\hline
\end{tabular}

Notes: *Different from MHNO phenotype $(p<0.05)$; Multivariate-adjusted model I was adjusted for sex; multivariate-adjusted model 2 was adjusted for sex, smoking status and alcohol consumption; multivariate-adjusted model 3 was adjusted for sex, smoking status, alcohol consumption, fasting glucose, total cholesterol, triglyceride and high-density lipoprotein cholesterol.

Abbreviations: NFS, NAFLD fibrosis score; MHNO, metabolically healthy non-obese; MUNO, metabolically unhealthy non-obese; MHO, metabolically healthy obese; MUO, metabolically unhealthy obese; OR, odds ratios.

Table 3 Subgroup Analysis Stratified by Sex for Advanced Fibrosis Using NFS (>0.676) Category

\begin{tabular}{|c|c|c|c|c|}
\hline & MHNO & MUNO & МнO & MUO \\
\hline & OR (95\% CI) & OR (95\% CI) & OR (95\% Cl) & OR (95\% Cl) \\
\hline \multicolumn{5}{|l|}{ Women } \\
\hline NFS category & I (reference) & $2.05(1.16-3.62)^{*}$ & $2.09(0.87-5.12)$ & $7.01(4.04-12.15) *$ \\
\hline Model 2 & I (reference) & $2.04(1.15-3.62)^{*}$ & $2.03(0.84-4.88)$ & $7.08(4.08-12.27)^{*}$ \\
\hline Model 3 & I (reference) & $1.60(0.81-3.17)$ & $2.08(0.84-5.14)$ & $4.70(2.53-8.72)^{*}$ \\
\hline \multicolumn{5}{|l|}{ Men } \\
\hline NFS category & I (reference) & $2.73(1.55-4.81)^{*}$ & $2.23(0.96-5.21)$ & $6.17(3.52-10.83)^{*}$ \\
\hline Model 2 & I (reference) & $2.79(1.57-4.94)^{*}$ & $2.21(0.94-5.21)$ & $6.10(3.47-10.73)^{*}$ \\
\hline Model 3 & I (reference) & $3.12(1.56-6.23)^{*}$ & $2.42(0.99-5.92)$ & $8.06(4.14-15.70)^{*}$ \\
\hline
\end{tabular}

Notes: *Different from MHNO phenotype $(p<0.05)$; multivariate-adjusted model 2 was adjusted for smoking status and alcohol consumption; multivariate-adjusted model 3 was adjusted for smoking status, alcohol consumption, fasting glucose, total cholesterol, triglyceride and high-density lipoprotein cholesterol.

Abbreviations: NFS, NAFLD fibrosis score; MHNO, metabolically healthy non-obese; MUNO, metabolically unhealthy non-obese; MHO, metabolically healthy obese; MUO, metabolically unhealthy obese; OR, odds ratios.

individuals with obesity had higher odds of developing NAFLD (OR for MHO: 3.55 [3.37-3.74]) and worsening of noninvasive fibrosis markers compared with those with normal weights (adjusted hazard ratio [HR] for BMI $\geq 30$ compared with BMI 18.5-22.9 was 3.52 [2.64-4.69]). The association was also stronger in women than in men (adjusted HR for BMI $\geq 30$ compared with BMI 18.5-22.9 in women was $5.76[5.23-6.34]$ and 2.87 [2.70-3.05] in men). ${ }^{37,38}$ Similarly, fatty liver was more prevalent in the MHO and MUO groups as well (adjusted OR for MUNO, MHO, and MUO: 3.75 [3.17-4.42], 7.52 [6.13-9.22], and 20.10 [16.20-24.90], respectively). ${ }^{39}$ A recent Taiwanese study also reported that both metabolic syndrome (OR, 3.19 [2.41-4.22]) and higher BMI (OR, 5.66 [3.91-8.19]) were positively associated with higher odds of fatty liver in the elderly. $^{40}$ 
Table 4 Univariate Analysis and Multivariate Analysis for Advanced Fibrosis Using FIB-4 (>2.67) According to Obese Phenotypes

\begin{tabular}{|l|l|l|l|l|}
\hline & MHNO & MUNO & MHO & MUO \\
\cline { 2 - 5 } & OR (95\% Cl) & OR (95\% CI) & OR (95\% Cl) & OR (95\% CI) \\
\hline FIB-4 category & I (reference) & $1.16(0.79-1.72)$ & $2.26(1.31-3.90)^{*}$ & $1.18(0.77-1.83)$ \\
Model I & I (reference) & $1.32(0.89-1.97)$ & $2.32(1.34-4.02)^{*}$ & $1.28(0.83-1.99)$ \\
Model 2 & I (reference) & $1.33(0.89-1.99)$ & $2.31(1.33-4.00)^{*}$ & $1.28(0.82-1.98)$ \\
Model 3 & I (reference) & $1.34(0.83-2.18)$ & $2.37(1.36-4.13)^{*}$ & $1.38(0.82-2.31)$ \\
\hline
\end{tabular}

Notes: *Different from MHNO phenotype $(p<0.05)$; multivariate-adjusted model I was adjusted for sex; multivariate-adjusted model 2 was adjusted for sex, smoking status and alcohol consumption; multivariate-adjusted model 3 was adjusted for sex, smoking status, alcohol consumption, fasting glucose, total cholesterol, triglyceride and high-density lipoprotein cholesterol.

Abbreviations: FIB-4, fibrosis-4 score; MHNO, metabolically healthy non-obese; MUNO, metabolically unhealthy non-obese; MHO, metabolically healthy obese; MUO, metabolically unhealthy obese; OR, odds ratios.

Table 5 Subgroup Analysis Stratified by Sex for Advanced Fibrosis Using FIB-4 (>2.67) Category

\begin{tabular}{|c|c|c|c|c|}
\hline & MHNO & MUNO & МHO & MUO \\
\hline & OR (95\% Cl) & OR $(95 \% \mathrm{Cl})$ & OR $(95 \% \mathrm{Cl})$ & OR $(95 \% \mathrm{CI})$ \\
\hline Women & & & & \\
\hline FIB-4 category & I (reference) & $1.45(0.82-2.56)$ & $3.84(1.86-7.95)^{*}$ & $1.54(0.83-2.85)$ \\
\hline Model 2 & I (reference) & I. $44(0.82-2.54)$ & $3.80(1.84-7.86)^{*}$ & $1.54(0.83-2.86)$ \\
\hline Model 3 & I (reference) & $1.60(0.82-3.12)$ & $3.70(\mid .78-7.7 I)^{*}$ & I.82 (0.87-3.77) \\
\hline Men & & & & \\
\hline FIB-4 category & I (reference) & $1.20(0.68-2.13)$ & $1.21(0.50-2.96)$ & $1.06(0.56-2.01)$ \\
\hline Model 2 & I (reference) & $1.22(0.69-2.18)$ & $1.20(0.49-2.94)$ & $1.05(0.55-1.99)$ \\
\hline Model 3 & I (reference) & $1.09(0.52-2.26)$ & $1.22(0.48-3.05)$ & I.82 (0.87-3.77) \\
\hline
\end{tabular}

Notes: *Different from MHNO phenotype ( $p<0.05$ ); multivariate-adjusted model 2 was adjusted for smoking status and alcohol consumption; multivariate-adjusted model 3 was adjusted for smoking status, alcohol consumption, fasting glucose, total cholesterol, triglyceride and high-density lipoprotein cholesterol.

Abbreviations: FIB-4, fibrosis-4 score; MHNO, metabolically healthy non-obese; MUNO, metabolically unhealthy non-obese; MHO, metabolically healthy obese; MUO, metabolically unhealthy obese; OR, odds ratios.

Conversely, Chen et al found that by using FIB-4 score to evaluate the risk for advanced fibrosis, higher BMI was not a risk factor (OR, $0.72[0.37-1.40])$ and Miyaaki et al stated that although there were more obese cases in the group with severe fibrosis, the association was not statistically significant (mild:severe $=67 \%: 78 \% ; p=0.23$ ) ${ }^{28}$ These findings were contrary to our study, which showed that the effect of obesity on more advanced fibrosis was significant. Furthermore, Gutiérrez-Grobe et al showed that advanced fibrosis was more prevalent in the MUO group than in the MHO group ( $28 \%$ vs $6.5 \%$, respectively; $p<0.05$ ), similar with our study results using NFS definition, but different when we used FIB-4 definition. ${ }^{41}$ In addition, Kim et al reported that in sex-stratified analysis, fibrosis progression was stronger in men than in women (HR for BMI $\geq 30 \mathrm{~kg} / \mathrm{m}^{2}$ compared with BMI $18.5-22.9 \mathrm{~kg} / \mathrm{m}^{2}$ in men was 4.83 [4.31-5.43] and 3.49 [2.90-4.20] in women), which was opposite from our finding. ${ }^{38}$ Their study composed of mainly premenopausal women, which estrogen may play a protective role, while ours are elder women with postmenopausal status. ${ }^{29,38,42}$

In our study, there are some differences in the results of advanced fibrosis assessed by NFS and FIB-4 scores (Tables 2 and 4). Several reasons might have accounted for this discrepancy. First, the prevalence rates of advanced fibrosis and the cutoff values affect the diagnostic performance of those tests. ${ }^{14,36}$ The best cutoff value is still debatable in the elderly and ethnic differences should take into account. Second, NFS formula included BMI and hyperglycemia, which are related directly to metabolic syndrome and obesity, while FIB-4 scores only included data on age, platelets, and liver enzymes. This may pose bias for detecting the association. Third, some patients with advanced fibrosis may not be detected and fall into the wrong category. ${ }^{43,44}$ Nevertheless, previous studies validated that NFS and FIB-4 scores best predict 
NAFLD advance fibrosis and are widely used in primary setting. ${ }^{36,44}$

Aging and visceral adiposity promotes metabolic abnormalities and decline insulin sensitivity. ${ }^{45,46}$ Excessive free fatty acids accelerate NAFLD pathogenesis and progression. ${ }^{47}$ Flux of free fatty acids from the adipose tissues into the liver can possibly induce lipid peroxidation and increasing proinflammatory cytokines, which can ultimately cause hepatic insult. ${ }^{48}$ With age-related decline in Kupffer cell deactivation of endotoxins and a slower, reduced ability to regenerate hepatocytes, fatty liver may progress to steatohepatitis and liver fibrosis. ${ }^{49}$ Estrogen is believed to have antioxidant and antisteatotic effects in the liver through sensitization of Kupffer cells to toxic stimuli and in driving the anti-inflammatory polarization of Kupffer cells. ${ }^{50,51}$ Perhaps loss of estrogen's protective role in NAFLD may contribute to fibrotic progression in postmenopausal women. In addition, women's adipose tissue distribution changes as estrogen declines in the elderly, shifting from gluteal-femoral area to abdominal region after menopause. ${ }^{52} \mathrm{~A}$ recent systemic review and meta-analysis concluded that women have lower risk of NAFLD than men in the general population but have higher risk of advanced fibrosis once NAFLD is established. The sex difference relationship is more evident among those aged over 50 years (RR, 1.56 $[1.36-1.80]) .{ }^{53}$ The exact mechanism of NAFLD in postmenopausal women is unclear, but sex hormones and fat distribution may play important roles in regulating steatohepatitis in the elderly. The present study, which demonstrated that NAFLD was associated with obesity and metabolic syndrome in the elderly, suggests that obesity phenotypes and metabolic health status are key features for further studies of NAFLD pathophysiology. In addition, obesity may be a more important contributor to fatty liver in elderly women than in men, perhaps because of the lack of estrogen protection and alterations in body composition as well as fat distribution. Further research is required to understand the sex-related difference in the elderly.

Our study indicates that obesity and metabolic syndrome have higher odds for advanced fibrosis in elderly individuals with NAFLD, and that using noninvasive fibrosis markers is more cost-effective and accessible for screening advanced fibrosis, especially in elderly women with obesity. Early management of liver fibrosis such as lifestyle modification, which includes changing dietary habits, weight reduction, and exercise can be encouraged.
Referral to gastrointestinal specialist for a more intense workup including FibroScan and acoustic radiation force impulse can also be suggested early on. Thus, progression of NAFLD-related complications such as cirrhosis and HCC may therefore be avoided.

Most previous studies focused on NAFLD prevalence, and the association of such prevalence with obesity phenotypes and metabolic health status is limitedly investigated, especially in the elderly population. To the best of our knowledge, this study is the first to explore the association between different obesity phenotypes and liver fibrosis score in elderly individuals with fatty liver in Taiwan. However, our study has several potential limitations. First, this study only focused on one center; thus, its generalizability to other population is uncertain. Second, no biopsy-proven pathologic diagnosis of NAFLD has been found, and the cutoff values for NFS and FIB-4 were based on previous studies that only included a small number of elderly individuals. ${ }^{54}$ Therefore, these values may not be appropriate for the elderly population. Furthermore, data on alcohol consumption, smoking status, past medical history, and current medication use were self-reported and may include past smokers and past drinkers; hence, these data may have some bias. Influence of exercise was not also considered. Moreover, agreement on a universally accepted definition of MHO, would improve the design of future studies and would facilitate better comparisons between studies. Larger studies with a more representative sample are required to obtain a more accurate description of the association between obesity phenotypes and NAFLD.

\section{Conclusion}

Metabolic syndrome and obesity were positively associated with a more advanced category of fibrosis according to NFS, which showed significant findings contrary to FIB-4 that only revealed a positive trend. Obesity seems to have a more detrimental effect than metabolic abnormalities per se on the severity of more advanced fibrosis in the elderly according to FIB-4 score, especially in women.

\section{Abbreviations}

ALT, alanine aminotransferase; ALT, alanine aminotransferase; BMI, body mass index; BP, blood pressure; CI, confidence interval; FIB-4, Fibrosis-4 score; HCC, hepatocellular carcinoma; HDL-C, high-density lipoprotein cholesterol; HR, hazard ratio; LDL, low-density 
lipoprotein cholesterol; MHNO, metabolically healthy non-obese; MHO, metabolically healthy obese; MUNO, metabolically unhealthy non-obese; MUO, metabolically unhealthy obese; NAFLD, nonalcoholic fatty liver disease; NASH, nonalcoholic steatohepatitis; NFS, NAFLD fibrosis score; OR, odds ratios; PPV, positive predictive value; $\mathrm{TC}$, total cholesterol; TG, triglyceride; T2DM, type 2 diabetes mellitus.

\section{Data Sharing Statement}

All data generated or analyzed during this study can be included in this published article and Supplementary Information Files.

\section{Ethics Approval and Informed Consent}

This is a non-interventional, observational and retrospective study with minimal risk for the participants. The study protocol was evaluated and approved by the institutional research board, Human Research Ethics Committee of Mackay Memorial Hospital (project research number 18MMHIS137), which confirmed that the study was conducted in accordance with the Declaration of Helsinki and that informed consent from the participants was not deemed necessary.

\section{Author Contributions}

Meng-Ting Tsou had full access to all of the data in the study and takes responsibility for the integrity of the data and the accuracy of the data analysis. Study concept and design: Yu-Shan Lee, Lee-Ching Hwang, Meng-Ting Tsou. Acquisition of data: Yu-Shan Lee, Meng-Ting Tsou. Analysis and interpretation of data: Yu-Shan Lee, Hsin-Yin Hsu, Lee-Ching Hwang, MengTing Tsou. Drafting of the manuscript: Yu-Shan Lee, Hsin-Yin Hsu. Critical revision of the manuscript for important intellectual content: Yu-Shan Lee, Lee-Ching Hwang, Meng-Ting Tsou. Statistical analysis: Yu-Shan Lee, Hsin-Yin Hsu. Study supervision: Lee-Ching Hwang, Meng-Ting Tsou.

All authors made substantial contributions to conception and design, acquisition of data, or analysis and interpretation of data; took part in drafting the article or revising it critically for important intellectual content; agreed to submit to the current journal; gave final approval of the version to be published; and agree to be accountable for all aspects of the work.

\section{Funding}

The authors disclosed no receipt of any financial support for the study, authorship of this article.

\section{Disclosure}

The authors report no conflicts of interest in this work.

\section{References}

1. Seto WK, Yuen MF. Nonalcoholic fatty liver disease in Asia: emerging perspectives. $J$ Gastroenterol. 2017;52(2):164-174. doi:10.1007/s00535-016-1264-3

2. Chen CH, Huang MH, Yang JC, et al. Prevalence and risk factors of nonalcoholic fatty liver disease in an adult population of Taiwan: metabolic significance of nonalcoholic fatty liver disease in nonobese adults. J Clin Gastroenterol. 2006;40(8):745-752. doi:10.1097/ 00004836-200609000-00016

3. Lin TJ, Lin CL, Wang CS, Liu SO, Liao LY. Prevalence of HFE mutations and relation to serum iron status in patients with chronic hepatitis $\mathrm{C}$ and patients with nonalcoholic fatty liver disease in Taiwan. World J Gastroenterol. 2005;11(25):3905. doi:10.3748/wjg. v11.i25.3905

4. Hung SC, Lai SW, Chen MC, Li PC, Lin KC. Prevalence and related factors of non-alcoholic fatty liver disease among the elderly in Taiwan. Eur Geriatr Med. 2013;4(2):78-81. doi:10.1016/j. eurger.2012.11.004

5. Kojima S, Watanabe N, Numata M, Ogawa T, Matsuzaki S. Increase in the prevalence of fatty liver in Japan over the past 12 years: analysis of clinical background. $J$ Gastroenterol. 2003;38 (10):954-961. doi:10.1007/s00535-003-1178-8

6. McPherson S, Hardy T, Henderson E, Burt AD, Day CP, Anstee QM. Evidence of NAFLD progression from steatosis to fibrosing-steatohepatitis using paired biopsies: implications for prognosis and clinical management. J Hepatol. 2015;62(5):1148-1155. doi:10.1016/j.jhep.2014.11.034

7. Singh S, Allen AM, Wang Z, Prokop LJ, Murad MH, Loomba R. Fibrosis progression in nonalcoholic fatty liver vs nonalcoholic steatohepatitis: a systematic review and meta-analysis of paired-biopsy studies. Clin Gastroenterol Hepatol. 2015;13(4):643-654. doi:10.1016/j.cgh.2014.04.014

8. Argo CK, Northup PG, Al-Osaimi AM, Caldwell SH. Systematic review of risk factors for fibrosis progression in non-alcoholic steatohepatitis. J Hepatol. 2009;51(2):371-379. doi:10.1016/j. jhep.2009.03.019

9. Lonardo A, Byrne CD, Caldwell SH, Cortez-Pinto H, Targher G. Global epidemiology of nonalcoholic fatty liver disease: meta-analytic assessment of prevalence, incidence, and outcomes. Hepatology. 2016;64(4):1388-1389. doi:10.1002/hep.28584

10. Kumar R, Priyadarshi RN, Anand U. Non-alcoholic fatty liver disease: growing burden, adverse outcomes and associations. $J$ Clin Transl Hepatol. 2020;8(1):76-86. doi:10.14218/JCTH.2019.00051

11. Said A, Ghufran A. Epidemic of non-alcoholic fatty liver disease and hepatocellular carcinoma. World J Clin Oncol. 2017;8(6):429-436. doi:10.5306/wjco.v8.i6.429

12. Ekstedt M, Hagstrom H, Nasr P, et al. Fibrosis stage is the strongest predictor for disease-specific mortality in NAFLD after up to 33 years of follow-up. Hepatology. 2015;61(5):1547-1554. doi:10.1 002/hep. 27368

13. Angulo P, Kleiner DE, Dam-Larsen S, et al. Liver fibrosis, but no other histologic features, is associated with long-term outcomes of patients with nonalcoholic fatty liver disease. Gastroenterology. 2015;149(2):389-397e310. doi:10.1053/j.gastro.2015.04.043 
14. Shah AG, Lydecker A, Murray K, et al. Comparison of noninvasive markers of fibrosis in patients with nonalcoholic fatty liver disease. Clin Gastroenterol Hepatol. 2009;7(10):1104-1112. doi:10.1016/j. cgh.2009.05.033

15. Angulo P, Hui JM, Marchesini G, et al. The NAFLD fibrosis score: a noninvasive system that identifies liver fibrosis in patients with NAFLD. Hepatology. 2007;45(4):846-854. doi:10.1002/hep.21496

16. Sterling RK, Lissen E, Clumeck N, et al. Development of a simple noninvasive index to predict significant fibrosis in patients with $\mathrm{HIV} / \mathrm{HCV}$ coinfection. Hepatology. 2006;43(6):1317-1325. doi:10.1002/hep.21178

17. Subasi CF, Aykut UE, Yilmaz Y. Comparison of noninvasive scores for the detection of advanced fibrosis in patients with nonalcoholic fatty liver disease. Eur J Gastroenterol Hepatol. 2015;27(2):137-141. doi:10.1097/MEG.0000000000000255

18. Mishra A, Younossi ZM. Epidemiology and natural history of non-alcoholic fatty liver disease. J Clin Exp Hepatol. 2012;2 (2):135-144. doi:10.1016/S0973-6883(12)60102-9

19. Fan JG, Saibara T, Chitturi S, et al. What are the risk factors and settings for non-alcoholic fatty liver disease in Asia-Pacific? J Gastroenterol Hepatol. 2007;22(6):794-800. doi:10.1111/j.14401746.2007.04952.x

20. Fan JG, Zhu J, Li XJ, et al. Fatty liver and the metabolic syndrome among Shanghai adults. $J$ Gastroenterol Hepatol. 2005;20 (12):1825-1832. doi:10.1111/j.1440-1746.2005.04058.x

21. Lonardo A, Mantovani A, Lugari S, Targher G. Epidemiology and pathophysiology of the association between NAFLD and metabolically healthy or metabolically unhealthy obesity. Ann Hepatol. 2020;19(4):359-366. doi:10.1016/j.aohep.2020.03.001

22. Ampuero J, Aller R, Gallego-Duran R, et al. The effects of metabolic status on non-alcoholic fatty liver disease-related outcomes, beyond the presence of obesity. Aliment Pharmacol Ther. 2018;48(11-12):1260-1270. doi:10.1111/apt.15015

23. Petta S, Eslam M, Valenti L, et al. Metabolic syndrome and severity of fibrosis in nonalcoholic fatty liver disease: an age-dependent risk profiling study. Liver Int. 2017;37(9):1389-1396. doi:10.1111/liv.13397

24. Lonardo A, Nascimbeni F, Mantovani A, Targher G. Hypertension, diabetes, atherosclerosis and NASH: cause or consequence? J Hepatol. 2018;68(2):335-352. doi:10.1016/j.jhep.2017.09.021

25. Chen SC, Tsai SP, Jhao JY, Jiang WK, Tsao CK, Chang LY. Liver fat, hepatic enzymes, alkaline phosphatase and the risk of incident type 2 diabetes: a prospective study of 132,377 adults. Sci Rep. 2017;7 (1):4649. doi:10.1038/s41598-017-04631-7

26. Sung KC, Wild SH, Byrne CD. Development of new fatty liver, or resolution of existing fatty liver, over five years of follow-up, and risk of incident hypertension. $J$ Hepatol. 2014;60(5):1040-1045. doi:10.1016/j.jhep.2014.01.009

27. Frith J, Day CP, Henderson E, Burt AD, Newton JL. Non-alcoholic fatty liver disease in older people. Gerontology. 2009;55(6):607-613. doi:10.1159/000235677

28. Miyaaki H, Ichikawa T, Nakao K, et al. Clinicopathological study of nonalcoholic fatty liver disease in Japan: the risk factors for fibrosis. Liver Int. 2008;28(4):519-524. doi:10.1111/j.1478-3231.2007.01614.x

29. Lonardo A, Nascimbeni F, Ballestri S, et al. Sex differences in nonalcoholic fatty liver disease: state of the art and identification of research gaps. Hepatology. 2019;70(4):1457-1469. doi:10.1002/ hep. 30626

30. Friedewald WT, Levy RI, Fredrickson DS. Estimation of the concentration of low-density lipoprotein cholesterol in plasma, without use of the preparative ultracentrifuge. Clin chem. 1972;18(6):499-502. doi:10.1093/clinchem/18.6.499
31. Martin SS, Blaha MJ, Elshazly MB, et al. Comparison of a novel method vs the Friedewald equation for estimating low-density lipoprotein cholesterol levels from the standard lipid profile. JAMA. 2013;310(19):2061-2068. doi:10.1001/jama.2013.280532

32. Hernaez R, Lazo M, Bonekamp S, et al. Diagnostic accuracy and reliability of ultrasonography for the detection of fatty liver: a meta-analysis. Hepatology. 2011;54(3):1082-1090. doi:10.1002/ hep. 24452

33. Pan WH, Flegal KM, Chang HY, Yeh WT, Yeh CJ, Lee WC. Body mass index and obesity-related metabolic disorders in Taiwanese and US whites and blacks: implications for definitions of overweight and obesity for Asians. Am J Clin Nutr. 2004;79(1):31-39. doi:10.1093/ajcn/79.1.31

34. Chen HY, Lu FH, Chang CJ, et al. Metabolic abnormalities, but not obesity per se, associated with chronic kidney disease in a Taiwanese population. Nutr Metab Cardiovasc Dis. 2020;30(3):418-425. doi:10.1016/j.numecd.2019.09.029

35. Eftekharzadeh A, Asghari G, Serahati S, et al. Predictors of incident obesity phenotype in nonobese healthy adults. Eur J Clin Invest. 2017;47(5):357-365. doi:10.1111/eci.12743

36. McPherson S, Hardy T, Dufour JF, et al. Age as a confounding factor for the accurate non-invasive diagnosis of advanced NAFLD fibrosis. Am J Gastroenterol. 2017;112(5):740-751. doi:10.1038/ajg.2016.453

37. Chang Y, Jung HS, Cho J, et al. Metabolically healthy obesity and the development of nonalcoholic fatty liver disease. Am J Gastroenterol. 2016;111(8):1133-1140. doi:10.1038/ajg.2016.178

38. Kim Y, Chang Y, Cho YK, Ahn J, Shin H, Ryu S. Metabolically healthy versus unhealthy obesity and risk of fibrosis progression in non-alcoholic fatty liver disease. Liver Int. 2019;39(10):1884-1894. doi:10.1111/liv.14184

39. Heianza Y, Arase Y, Tsuji H, et al. Metabolically healthy obesity, presence or absence of fatty liver, and risk of type 2 diabetes in Japanese individuals: Toranomon hospital health management center study 20 (TOPICS 20). J Clin Endocrinol Metab. 2014;99 (8):2952-2960. doi:10.1210/jc.2013-4427

40. Chen TP, Lai M, Lin WY, Huang KC, Yang KC. Metabolic profiles and fibrosis of nonalcoholic fatty liver disease in the elderly: a community-based study. $J$ Gastroenterol Hepatol. 2020;35 (9):1636-1643. doi:10.1111/jgh.15073

41. Gutierrez-Grobe Y, Juarez-Hernandez E, Sanchez-Jimenez BA, et al. Less liver fibrosis in metabolically healthy compared with metabolically unhealthy obese patients with non-alcoholic fatty liver disease. Diabetes Metab. 2017;43(4):332-337. doi:10.1016/j. diabet.2017.02.007

42. Ballestri S, Nascimbeni F, Baldelli E, Marrazzo A, Romagnoli D, Lonardo A. NAFLD as a sexual dimorphic disease: role of gender and reproductive status in the development and progression of nonalcoholic fatty liver disease and inherent cardiovascular risk. $A d v$ Ther. 2017;34(6):1291-1326. doi:10.1007/s12325-017-0556-1

43. Ishiba H, Sumida Y, Tanaka S, et al. The novel cutoff points for the FIB4 index categorized by age increase the diagnostic accuracy in NAFLD: a multi-center study. $J$ Gastroenterol. 2018;53 (11):1216-1224. doi:10.1007/s00535-018-1474-y

44. Patel YA, Gifford EJ, Glass LM, et al. Identifying nonalcoholic fatty liver disease advanced fibrosis in the veterans health administration. Dig Dis Sci. 2018;63(9):2259-2266. doi:10.1007/s10620-018-5123-3

45. Hamaguchi M, Kojima T, Takeda N, et al. The metabolic syndrome as a predictor of nonalcoholic fatty liver disease. Ann Intern Med. 2005;143(10):722-728. doi:10.7326/0003-4819-143-10-20051115000009 
46. Lonardo A, Suzuki A. Sexual dimorphism of NAFLD in adults. Focus on clinical aspects and implications for practice and translational research. $J$ Clin Med. 2020;9(5):1278. doi:10.3390/ jcm9051278

47. Friedman SL, Neuschwander-Tetri BA, Rinella M, Sanyal AJ. Mechanisms of NAFLD development and therapeutic strategies. Nat Med. 2018;24(7):908-922. doi:10.1038/s41591-018-0104-9

48. Farrell GC, Larter CZ. Nonalcoholic fatty liver disease: from steatosis to cirrhosis. Hepatology. 2006;43(2 Suppl 1):S99-S112. doi:10.1002/hep.20973

49. Gan L, Chitturi S, Farrell GC. Mechanisms and implications of age-related changes in the liver: nonalcoholic Fatty liver disease in the elderly. Curr Gerontol Geriatr Res. 2011;2011:831536. doi: $10.1155 / 2011 / 831536$

50. Gutierrez-Grobe Y, Ponciano-Rodríguez G, Ramos MH, Uribe M, Méndez-Sánchez N. Prevalence of non alcoholic fatty liver disease in premenopausal, posmenopausal and polycystic ovary syndrome women. The role of estrogens. Ann Hepatol. 2010;9(4):402-409. doi:10.1016/S1665-2681(19)31616-3
51. Della Torre S. Non-alcoholic fatty liver disease as a canonical example of metabolic inflammatory-based liver disease showing a sex-specific prevalence: relevance of estrogen signaling. Front Endocrinol (Lausanne). 2020;11:572490.

52. Karastergiou K, Smith SR, Greenberg AS, Fried SK. Sex differences in human adipose tissues - the biology of pear shape. Biol Sex Differ. 2012;3(1):13. doi:10.1186/2042-6410-3-13

53. Balakrishnan M, Patel P, Dunn-Valadez S, et al. Women have a lower risk of nonalcoholic fatty liver disease but a higher risk of progression vs men: a systematic review and meta-analysis. Clin Gastroenterol Hepatol. 2021;19(1):61-71e15. doi:10.1016/j. cgh.2020.04.067

54. Pitisuttithum P, Chan WK, Piyachaturawat P, et al. Predictors of advanced fibrosis in elderly patients with biopsy-confirmed nonalcoholic fatty liver disease: the GOASIA study. BMC Gastroenterol. 2020;20(1):88. doi:10.1186/s12876-020-01240-z

\section{Publish your work in this journal}

Diabetes, Metabolic Syndrome and Obesity: Targets and Therapy is an international, peer-reviewed open-access journal committed to the rapid publication of the latest laboratory and clinical findings in the fields of diabetes, metabolic syndrome and obesity research. Original research, review, case reports, hypothesis formation, expert opinion and commentaries are all considered for publication. The manuscript management system is completely online and includes a very quick and fair peer-review system, which is all easy to use. Visit http://www.dovepress.com/testimonials.php to read real quotes from published authors. 